\title{
Exotic Nuclei in South America
}

Cite as: AIP Conference Proceedings 1265, 27 (2010); https://

doi.org/10.1063/1.3480185

Published Online: 05 August 2010

R. Lichtenthäler, A. Lépine-Szily, V. Guimarães, P. N. de Faria, D. R. Mendes, K. C. C. Pires, V. Morcelle, A. Barioni, M. C. Morais, R. Pampa Condori, M. Assunção, A. M. Moro, M. Rodríguez-Gallardo, and A. Arazi

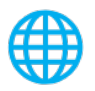

\section{Lock-in Amplifiers $\underset{\text { Watch }}{>}$
up to $600 \mathrm{MHz}$}

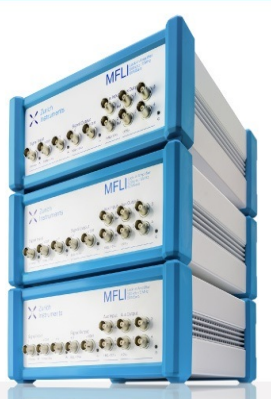




\title{
Exotic Nuclei in South America
}

\author{
R. Lichtenthäler ${ }^{a}$, A. Lépine-Szily ${ }^{a}$, V. Guimarães ${ }^{a}$, P.N. de Faria ${ }^{a}$, D.R. \\ Mendes Jr. ${ }^{a}$, K.C.C. Pires ${ }^{a}$, V. Morcelle ${ }^{a}$, A. Barioni ${ }^{a}$, M.C. Morais ${ }^{a}$, R. \\ Pampa Condori, ${ }^{a}$ M. Assunção ${ }^{e}$, A.M. Moro ${ }^{b}$, M. Rodríguez-Gallardo ${ }^{b, c}$
}

\author{
,A. $\operatorname{Arazi}^{d}$ \\ ${ }^{a}$ Instituto de Fisica da USP, São Paulo, Brazil, C.P. 66318, 05314-970 \\ ${ }^{b}$ Departamento de FAMN, Universidad de Sevilla, Apdo. 1065, E-41080, Sevilla, Spain \\ ${ }^{c}$ Instituto de Estructura de la Materia, CSIC, Serrano 123, E-28006 Madrid, Spain \\ ${ }^{d}$ Laboratorio TANDAR, Comision Nacional de Energia Atomica, Argentina \\ ${ }^{e}$ Departamento de Ciencias Exatas e da Terra, Unifesp, Campus de Diadema, São Paulo, Brazil
}

\begin{abstract}
The Radioactive Ion Beams in Brasil (RIBRAS) is described. Experiments using radioactive secondary beams of light rare isotopes such as ${ }^{6} \mathrm{He},{ }^{7} \mathrm{Be},{ }^{8} \mathrm{Li}$ on several targets have been performed and the results are presented.
\end{abstract}

Keywords: Rare isotopes, Radioactive Beams, Elastic Scattering, neutron halo, transfer reactions

PACS: 25.60-t,25.60.Bx

The study of reaction induced by secondary beams of rare isotopes is one of the most active fields in Nuclear Physics nowadays. Nuclei far from the line of beta stability are called exotic nuclei and present unusual features compared to the stable isobars. Neutron rich nuclei like ${ }^{6,8} \mathrm{He}$ present a cluster like structure formed by an alpha core and a "halo" of neutrons which extends over large distances from the core and with densities much lower than the normal nuclear matter. The weakly bound neutrons enhances the total reaction cross sections of those exotic nuclei and the possible presence of such rare isotopes in astrophysical sites like supernovas explosions and the Big-Bang nucleosynthesis could have consequences in the formation of the stable known elements.

RIBRAS is presently the only experimental equipment in South America capable of producing secondary beams of rare isotopes. It consists of two superconducting solenoids, presently installed in one of the beam lines of the 8 MV Pelletron Tandem

CP1265, VIII Latin American Symposium on Nuclear Physics and Applications edited by R. Alcaron, H. F. Arellano, P. L. Cole, and A. J. Kreiner

(C) 2010 American Institute of Physics 978-0-7354-0814-2/10/\$30.00 
accelerator of the University of São Paulo. The exotic nuclei are produced in the collision between the primary beam of the Pelletron Acceleratorand the primary target, placed just before the first solenoid (see figure I). The first solenoid focus the secondary beam on a secondary target where the reaction of interest takes place. The ${ }^{7} \mathrm{Li}$ of 3-5 MeV/A and a ${ }^{9} \mathrm{Be}\left(\sim 12 \mu_{\mathrm{m}}\right)$ are the standard primary beam and primary target to produce ${ }^{6} \mathrm{He}$ and ${ }^{8} \mathrm{Li}$ secondary beams beams via the one proton stripping ${ }^{9} \mathrm{Be}\left({ }^{7} \mathrm{Li},{ }^{6} \mathrm{He}\right)$ and one neutron pickup ${ }^{9} \mathrm{Be}\left({ }^{7} \mathrm{Li},{ }^{8} \mathrm{Li}\right)$ reactions. A ${ }^{3} \mathrm{He}$ gas primary target is used to produce the ${ }^{8} \mathrm{~B}$ and ${ }^{7} \mathrm{Be}$ beams by the ${ }^{3} \mathrm{He}\left({ }^{6} \mathrm{Li},{ }^{8} \mathrm{~B}\right)$ and ${ }^{3} \mathrm{He}\left({ }^{7} \mathrm{Li},{ }^{7} \mathrm{Be}\right)$ reactions. The primary beam cross the primary target (see label (1) in Figure 1) and is collected in the Faraday cup (3). The secondary particles produced in the primary target are collected by the first solenoid within a cone between $2^{\circ} \leq \theta \leq 6^{\circ}$ defined by the Faraday cup and the collimator (2). The acceptance solid angle of the system is $30 \mathrm{msr}$. The first solenoid makes a magnetic-rigidity selection to focus the secondary beam in the secondary target position (7). Unwanted secondary particles are stopped in the blocker (5) and collimator (6). A second identical solenoid is mounted after the target (7) and will allow an additional filtering improving the purity of the secondary beams. Presently we operate only with the first solenoid.

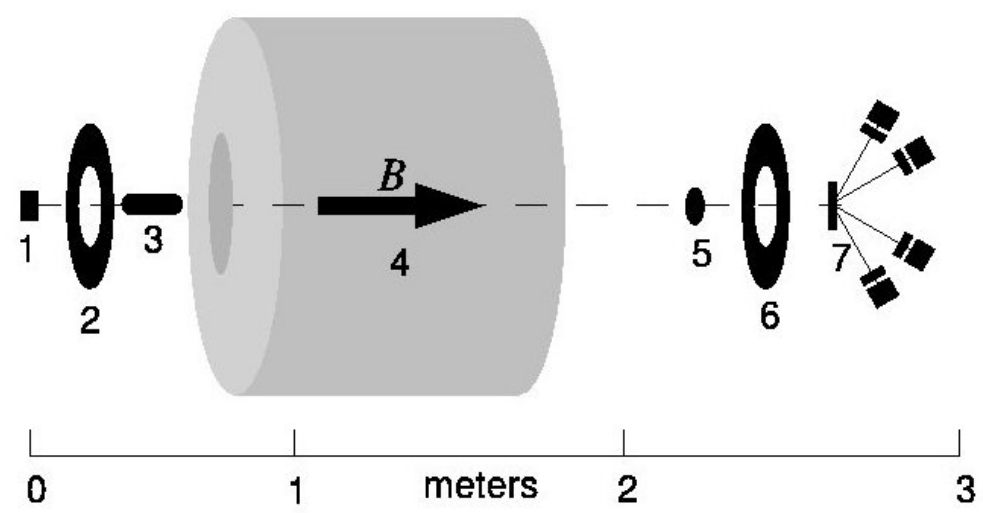

FIGURE 1. Scheme of the first solenoid RIBRAS system. -1-primary target,

2-collimator, 3-Farady cup, 4-solenoid, 5-unwanted-beam blocker, 6-collimator, 7-secondary target and detectors. 
This system allows the production of beams of the light nuclei mentioned above with intensities of $10^{4}$ to $10^{6} \mathrm{pps}$ and energies in the range 1-5 MeV/A.

The research performed at RIBRAS consists basically of elastic scattering and nucleon transfer reactions studies at low energies, using light exotic beams such as ${ }^{6} \mathrm{He},{ }^{8} \mathrm{Li}$ and ${ }^{7} \mathrm{Be}$. The elastic scattering allows the study of the interacting potential and the total reaction cross section. We have been developing this program using the medium-heavy target ${ }^{120} \mathrm{Sn}$ [2], medium mass targets such as ${ }^{58} \mathrm{Ni}$ and ${ }^{51} \mathrm{~V}$ [3] and lighter targets such as ${ }^{27} \mathrm{Al}[4],{ }^{12} \mathrm{C}[5,6]$ and ${ }^{9} \mathrm{Be}[7,8]$. The sistematic study using targets of different atomic numbers allows the investigation of the interplay between the Coulomb and nuclear interactions in the collision. Due to the fact that the exotic nuclei are weakly bound, the breakup and the neutron transfer reactions become important channels even at energies below the Coulomb barrier, strongly coupled with the elastic channel. As a consequence, the usual optical potentials fail to reproduce the elastic angular distributions and the coupling to the continuum has to be considered explicitly via CDCC calculations $[8,15]$. The presence of a long range imaginary potential is a feature present in the scattering of ${ }^{6} \mathrm{He}$ on heavy targets and is probably due to the projectile breakup in the Coulomb field of the target.

The total reaction cross section of exotic projetiles presents an enhancement compared to stable isobar projectiles in identical heavy targets $[2,13]$.

To compare the reaction cross sections of different systems at different energies, it is convenient to rescale the cross sections and energies in order to overcome trivial effects due to the different sizes and energies with respect to the Coulomb barrier $[10,11]$

For lighter systems such as ${ }^{6} \mathrm{He}+{ }^{27} \mathrm{Al},{ }^{6} \mathrm{He}+{ }^{12} \mathrm{C}$ and ${ }^{6} \mathrm{He}+{ }^{9} \mathrm{Be}[7,8]$ situation is still inconclusive [12]. There are some indications that the enhancement observed in ${ }^{6} \mathrm{He}+$ heavy targets could be smaller for lighter systems such as the ${ }^{6} \mathrm{He}+{ }^{27} \mathrm{Al}[4]$ and ${ }^{8} \mathrm{~B}+{ }^{12} \mathrm{C}$ [6], although a larger reduced reaction cross section has been obtained in some analysis [9].

Another interesting field opened by the advent of exotic secondary beams is the spectroscopic study of nuclei out of the line of stability and its consequences in reactions of astrophysical interest. The proton transfer ${ }^{12} \mathrm{C}\left({ }^{8} \mathrm{Li},{ }^{9} \mathrm{Be}\right){ }^{11} \mathrm{~B}$ reaction has been measured at RIBRAS [5,6]. As the vertex $\left\langle\left.{ }^{11} B\right|^{12} C\right\rangle$ is known, this reaction provides information of the $<\left.^{8} \mathrm{Lil}\right|^{9} \mathrm{Be}>$ spectroscopic factor, which normalizes the radiative capture ${ }^{8} \mathrm{Li}+p \rightarrow{ }^{9} \mathrm{Be}$ cross section. 


\section{ACKNOWLEDGMENTS}

The authors thank the Fundação de Amparo à Pesquisa do Estado de São Paulo (FAPESP) and the Conselho Nacional de Desenvolvimento Científico e Tecnológico (CNPq) for financial support.

\section{REFERENCES}

1. R. Lichtenthäler et al., Eur. Phys. J. A 25,s01,733 (2005) and Nucl. Phys. News 15, 25 (2005).

2. "The Scientific program at RIBRAS, R. Lichtenthäler et al., AIP Conference proceedings 1139, 76 (2009). http://scitation.aip.org/dbt/dbt.jsp?KEY=APCPCS\&Volume=1139\&ssue $=1$

3. V. Morcelle, Master Thesis, Instituto de Física da USP (2007)

4. E. Benjamin et al., Physics Letters B647,30,(2007)

5. A. Barioni, PhD Thesis, Instituto de Física da USP (2009)

6. A. Barioni et al. Phys. Rev. C 80, 034617(2009)

7. K.C.C. Pires, The $6 \mathrm{He}+9 \mathrm{Be}$ scattering, $\mathrm{PhD}$ Thesis, in preparation (see ref. 8)

8. http://cdsweb.cern.ch/record/1238174? ln=pt

9. M. Rodríguez-Gallardo et al, Phys.Rev.C77, 064609(2008)

10. J.M.B. Shorto et al. Phys. Lett. B678(2009)77

11. P.R.S. Gomes et. al. Phys. Lett. B601, 20(2004)

12.V. Guimarães et. al. Phys. Rev. C75, 054602(2007)

13. Faria, PhD Thesis, Instituto de Física da USP (2008) 